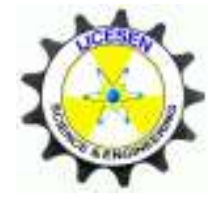

Copyright (C) IJCESEN
International Journal of Computational and

Experimental Science and $\boldsymbol{E N g i n e e r i n g}$

(IJCESEN)

Vol. 7-No.3 (2021) pp. 156-163

http://dergipark.org.tr/en/pub/ijcesen

Research Article

\title{
The Prediction of Chiral Metamaterial Resonance using Convolutional Neural Networks and Conventional Machine Learning Algorithms
}

\author{
Aybike URAL YALÇIN ${ }^{1}$, Zeynep Hilal KİLIMCí2,* \\ ${ }^{1}$ Doğuş University, Engineering Faculty, Department of Mechanical Engineering, 34775, İstanbul-Turkey \\ Email: aural@dogus.edu.tr ORCID: 0000-0002-3923-1821 \\ ${ }^{2}$ Kocaeli University, Technology Faculty, Department of Information Systems Engineering, 41001, Kocaeli-Turkey \\ * Corresponding Author Email: zeynep.kilimci@ kocaeli.edu.tr ORCID: 0000-0003-1497-305X
}

\section{$\underline{\text { Article Info: }}$}

DOI: $10.22399 /$ ijcesen. 973726

Received : 22 July 2021

Accepted : 29 November 2021

\section{Keywords:}

Chiral metamaterials

Convolutional neural network

Deep learning

Machine learning

Microwave resonance

\begin{abstract}
:
Electromagnetic resonance is the most important distinguishing property of metamaterials to examine many unusual phenomena. The resonant response of metamaterials can depend many parameters such as geometry, incident wave polarization. The estimation and the design of the unit cells can be challenging for the required application. The research on resonant behavior can yield promising applications. We investigate the resonance frequency of the chiral resonator as a unit of chiral metamaterial employing both traditional machine learning algorithms and convolutional deep neural networks. To our knowledge, this is the very first attempt on chiral metamaterials in that comparing the impact of various machine learning algorithms and deep learning model. The effect of geometrical parameters of the chiral resonator on the resonance frequency is studied. For this purpose, convolutional neural networks, support vector machines, naive Bayes, decision trees, random forests are employed for classification of resonance frequency. Extensive experiments are performed by varying training set percentages, epoch sizes, and data sets. Experiment results demonstrate that the usage of convolutional neural networks is superior in terms of prediction performance of chiral metamaterial resonance compared to the other techniques with $58.29 \%$ of accuracy on dataset 1 , and $68.77 \%$ of accuracy on dataset 2 .
\end{abstract}

\section{Introduction}

The chirality refers to a structural property of an object that cannot be superimposed on its mirror image [1]. The existence of chirality in nature at macro and molecular scale lead to a wide variety of research. Despite the natural phenomenas [2], artificially made materials called metamaterials can exhibit chiral properties. Metamaterials are composed of periodically arranged resonant elements, unit cells, which show unnatural electromagnetic and optical properties, such as negative refraction, which are not seen in naturally occurring materials $[3,4]$. Various chiral resonators are used to build chiral metamaterials such as helical wires, chiral split ring resonators (SRR) [5], gammadions [4] or cross-wire [6] structures. The optical response of chiral metamaterials is studied also in terahertz regime [7-10]. Some designs are proposed to avoid bianisotropic effects [11-12].
Omega shaped particles are another building blocks of metamaterials for the realization of the negative index which is experimentally verified [13-14]. Transmission properties of metamaterials formed by omega shaped inclusions are investigated in [15]. It is shown that the periodic arrays can be employed in stop/pass band applications due to the magnetoelectric resonances. Another artificial chiral object is called a canonical spiral replacing a helix in order to simplify the electromagnetic analysis [16]. In [17], an analytical antenna model is used to analyze such chiral scatterers. The canonical spiral is designed for chiral metamaterials where independently the linear polarization of the incident wave can be radiated as a circularly polarized wave [18]. As a consequence of the design, a particular helicity is completely transparent for circular electromagnetic waves of the same helicity [18]. The canonical spirals are capable also in cloaking application [19]. 
In this work, we design a metamaterial structure given a desired chiral response or even to simply predict the trend in chiral response as the structure transforms. The electromagnetic response of canonical spiral shaped chiral resonators in the microwave regime will be examined by a proposed method based on both machine learning algorithms and deep learning methodology. The application of deep learning algorithms are rather popular in recent years in different research fields such as image processing, natural language processing, speech recognition, video processing, sentiment analysis, text classification, computer vision, pattern recognition, and machine translation. Furthermore, deep neural network models are also utilized in various research domains beyond computer science such as physics with various subfields like material science, chemistry, laser physics, particle physics, quantum mechanics, and microscopy [20-26]. Deep learning networks applied in many studies to the inverse engineering to reveal the metamaterials response in optics and acoustics [27-30]. For the purpose of eliminating disadvantages of conventional machine learning algorithms, deep learning methodology is preferred by the researches in many fields because of its superior performance. In other words, the main reason behind the choice of deep learning models by the researchers both better representation of features, predictions, and results when compared with traditional machine learning algorithms. Deep learning models are mostly employed to ensure automatic feature extraction procedure thereby training complex features with minimal external support to achieve meaningful representation of data through deep neural networks. Besides automatic feature extraction, deep learning methods are also used for the purpose of classification tasks in many fields. Due to both excellent performance of deep learning models in the state-of-the-art studies and the lack of implementation of deep learning models on optical chiral metamaterials subfield, we concentrate on Convolutional Neural Network (CNN) as deep learning model for the purpose of eliminating this deficiency in this study, in addition to machine learning algorithms.

In the present work, the resonance frequency of the chiral structure as a unit of chiral metamaterial is studied using both traditional machine learning algorithms and convolutional deep neural networks. The main objective of this study is the prediction of the resonance response of the canonical spiral through the proposed model. For this purpose, convolutional neural networks, support vector machines, naive Bayes, decision trees, random forests are used for frequency selective classification. The novelty of this study is to investigate the impact of the most popular deep learning method, CNN, which is not implemented yet on the chiral metamaterial design. To the best of our knowledge, this is the very first study on chiral metamaterials in that comparing the impact of various machine learning algorithms and deep learning model.

The paper is organized as follows: In section 2, machine learning algorithms and convolutional neural network employed in this study is introduced. In section 3, we describe the chiral resonator and the numerical model from which we gathered the data and the proposed framework. The section 4 discusses the results and then we conclude the paper.

\section{Models}

In this section, methods used in this work are briefly presented.

\subsection{Naive Bayes Algorithm (NB)}

Naive Bayes is a well-known and mostly employed classification model for both two-class (binary) and multi-class classification problems. The naive Bayes algorithm is based upon independency of features in the data set that is base of Bayes's theorem. It is easy to construct and not complex which makes it especially beneficial for huge data sets. In spite of its simpleness, the Naive Bayes algorithm works well and presents superior classification performances compared to more complicated classification models. There are different event models for NB algorithm such as Gaussian naive Bayes, Multinomial naïve Bayes, Bernoulli naive Bayes, etc. Gaussian NB model is the easiest way to forecast the distribution of the data set because it predicts just the standard deviation and the mean of training data set. In this work, we focus on the Gaussian naive Bayes method [31-36].

\subsection{Support Vector Machine (SVM)}

Support vector machine (SVM) is employed as a supervised learning method that is used for classification and regression problems. The main purpose of support vector machine method is to discover a hyperplane in an $\mathrm{N}$-dimensional space that plainly categorizes the points of data set. However, support vector machine is responsible for finding a plane with the maximum space between data points of both categories among many potential hyperplanes for the purpose of dividing the two classes. This procedure is called margin maximization that facilitates the classification task for unseen instances with more confidence. Furthermore, SVM is also capable to implement 
non-linear classification task in addition to linear classification which is called kernel trick, implicitly mapping their inputs into high-dimensional spaces. According to data set distribution, there are too many kernel tricks such as linear, polynomial, radial basis function, sigmoid when SVM is constructed. In this work, each version is implemented and polynomial is selected as the best [33-40].

\subsection{Decision Tree (DT)}

Decision tree method is one of the predicting models employed in machine learning. Decision trees are also popular machine learning algorithms in terms of their comprehensibility and simplicity. The main objective of decision trees is to construct a model that predicts a target value (class) based upon different inputs. A decision tree is evaluated as a predictive model with branches and leaves. Here, observations are the branches while target value of related observation is represented as leaves. In classification trees, class labels are demonstrated with leaves and association of the features are indicated as branches. In other words, a tree is constructed by dividing the source data set, composing the root node of the tree, into subsets (children). The dividing procedure is implemented with a set of splitting rules based upon features of classification. When this procedure is repeated for each derivate subset, it is called as recursion. Recursion is finished when target variable has same results at a node for each subset. This widely accepted approach for decision trees is known as top-down induction that signs the greedy algorithm. As a summary, decision trees ensure the consolidation of computational and mathematical methods to obtain the classification of the dedicated data set [33,41-43].

\subsection{Random Forest (RF)}

Random forest is based on a large number of decision trees that constructs a community decision system. Each decision tree in a community demonstrates the class forecast and final decision is determined by voting according to majority. Random forests are proposed in order to eliminate the over-fitting problems of decision trees. The basic idea behind of random forest is constructing the common decision of different models which exhibits more successful classification success compared to the only one classifier. Here, two main concepts are important. It is expected that each individual tree is constructed through low correlated models in a random forest and high classification success. The main reason behind of this expectation is to interfere individual errors of each tree from each other by reducing relation between models. In this work, the number of individual trees is set to 25 due to its superior performance compared to $10,50,75$, and 100 of trees [34-35,44-46].

\subsection{Convolution Neural Network (CNN)}

Convolutional neural network is a type of deep neural networks, mostly applied especially in image processing field. CNNs exhibits notable classification results in natural language processing, financial time series, video processing and are also popular in these domains. The name this network model comes from the series of mathematical process named as convolution and is a type of customized linear operations. $\mathrm{CNN}$ is mainly constructed with input layer, multiple hidden layers, and output layer. Hidden layers of $\mathrm{CNN}$ are comprised from the sets of convolutional layers that rely on a convolution instead of matrix multiplication in at least one of their layers. CNN methodology includes a set of convolutional layers intertwined with pooling layers, followed up several fully connected layers. During this procedure, the most significant layer is the convolution layer that implement a filter of convolution to input in order to attain a feature map of input data. In order to get multiple features, multiple filters are carried out during training process and filters are capable to define the context of an investigated problem. After that, convolution process is implemented to acquire feature maps which indicates dependencies among features, local features of data set. Then, pooling layers that are intertwined with convolutional layers are acted to decrease the number of instances in each feature map and keep the most significant information about data. Through down sampling characteristic, the decrease in training time of the system and dimension reduction of data set are provided. There are several kinds of pooling layers such as max pooling, average pooling, global max pooling, global average pooling. In this work, maximum pooling is utilized which is generally also employed in literature studies. Actually, feature extraction is performed through convolution and pooling layers until this step. After, the output of pooling layers is converted $\mathrm{nx} 1$ dimensional vector in order to feed fully connected layers which is called flattening. Then, final decision of the system is determined by the help of fully connected layers. In CNN architecture like other deep neural networks, there are too many methods to avoid over-fitting challenge such as regularization models, early stopping criteria. In this study, we use dropout, L2 regularizator, and early stopping criteria for this purpose [33, 35, 47-55]. 


\section{Proposed Framework}

\subsection{Data Preparation}

In this part, data preparation is introduced. Figure 1 shows the schematic view of the canonical spiral which is composed of a torus with a gap $g=$ $0.9 \mathrm{~mm}$ and two rods residing from edges of the gap in opposite directions perpendicular to the plane of the torus. The outer radius of torus is $r_{\text {out }}=2 \mathrm{~mm}$ and torus thickness $r=0.8 \mathrm{~mm}$; two legs are of length $l=2 \mathrm{~mm}$. The thickness of the torus is kept fixed, while the gap size, radius of the torus and the length of the legs is varying. In data gathering process, three gap sizes are used between $0.9 \mathrm{~mm}$ and $1.1 \mathrm{~mm}$. For each gap size, we are changing the length of the rods and the radius of the torus between $2 \mathrm{~mm}$ and $3 \mathrm{~mm}$ with a step size $0.1 \mathrm{~mm}$.
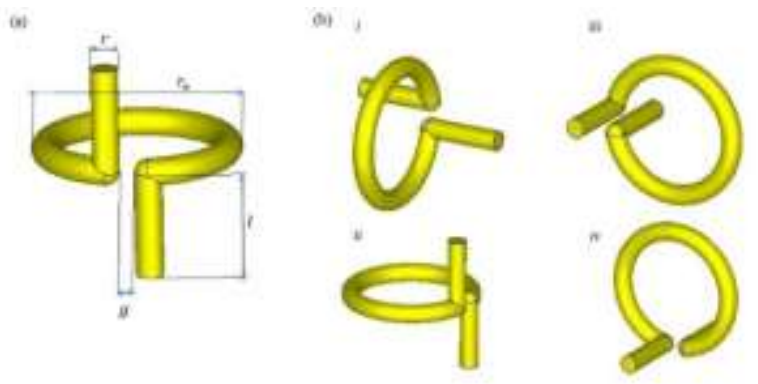

Figure 1. The canonical spiral has gap g, length of the rods $l$, thickness $r$, and outer radius of the torus is $r_{0}$.

Four orientations $i$-iv of the canonical spiral with respect to $E, B$ field and the propagation direction of the incident plane wave.

The structure is taken as perfect electric conductor (PEC). The embedding environment is taken as air. A plane wave travelling along $+\mathrm{x}$-axis is incident on the structure where the electric field is linearly polarized along $y$-axis, and the magnetic field is along z-axis. The resonant frequency response of the canonical spiral is obtained analyzing the transmission $\left(\mathrm{S}_{21} \mathrm{~dB}\right)$ spectrum between $5-$ $11 \mathrm{GHz}$. The four possible orientations of the canonical spiral with respect to the incident EM wave is examined and their resonance response is used in classification. The simulations are computed in CST Microwave studio software, which uses finite element methods, in frequency domain solver. The EM wave is excited defining ports and relevant boundary conditions

\subsection{Model}

In this work, we focus on the estimating resonance frequency of chiral metamaterial by employing both traditional machine learning models and a deep learning algorithm. For this purpose, naive Bayes (NB), support vector machine (SVMs), decision trees (DTs), random forests (RFs) are evaluated as machine learning algorithms and convolutional neural networks (CNNs) are appraised as a deep learning model in order to estimate of chiral metamaterial resonance. To demonstrate the efficiency of proposed design, we collect two data sets (dataset1, dataset2) that contain 1,210 and 2,057 instances, respectively. The number of features and class labels are the same for both data sets. Features evaluated in the training procedure are length of the rods, outer radius of torus, thickness of torus, gap, orientation in millimeters. Resonance frequency is located as class label in giga hertz. The main objective of our study is assigning resonance frequency of unseen/unlabeled instances in the data set by training model through machine learning algorithms and a deep learning model. In order to prepare data set for training procedure, there are some adaptations by converting categorical values into numerical ones such as orientation attribute. Moreover, class label is scaled per 0.05 precision starting from 0 to 1 corresponding to canonical spiral designs with a resonance frequency classes $5-$ $11 \mathrm{GHz}$ with a stepsize 0.05 . After that, the data set is randomly splitted into training and test sets by varying training set size as $80,50,30,10$. The remainings are carried out as test set percentages. To achieve a reliable prediction, the holdout process is recurred 10 times and an overall accuracy is calculated by taking averages of each iteration. In the tables, the following abbreviations are employed. Ts: Training set size, NB: Naive Bayes algorithm, SVM: Support vector machine, DT: Decision tree, RF: Random Forest algorithm, CNN: Convolutional neural network. The best classification results are exhibited in boldface in the tables. Furthermore, accuracy is appraised as an evaluation metric. In Figure 2, the proposed framework is presented.

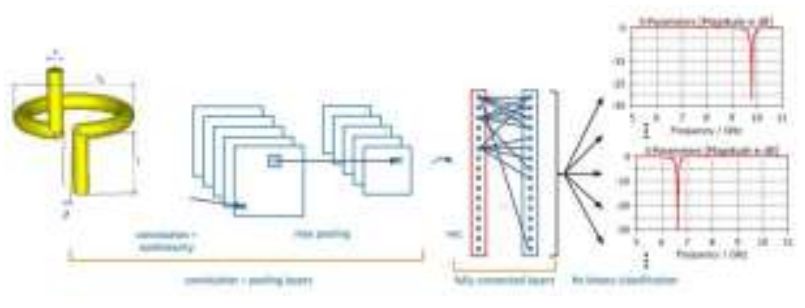

Figure 2. The canonical spiral geometrical parameters and its orientation given as input to the convolution plus pooling layers, as output we get the resonance frequency which corresponds to a dip in the transmission spectrum.

\section{Experiment Results}


Firstly, the effect of conventional machine learning algorithms is investigated on frequency prediction of chiral metamaterials. Then, convolutional neural networks are evaluated in order to observe the impact a deep learning model on this classification task. Table 1 and Table 3 demonstrates the classification accuracies of machine learning models and deep learning model on both data sets in terms of training set percentages. First of all, it is clearly observed in Table 1 and Table 3 that the usage of ts 80 boosts the classification accuracy of each model. The poorest classification performance is exhibited at ts 10 because the data set allocated for training is very small. As the training set percentage increases, classification accuracy of the system is enhanced as expected. For this reason, the setting of training set percentage to 80 is an effective way when system performance is considered.

In Table 1, the resonance frequency classification results of each algorithm are presented on dataset1 by varying training set sizes. When the average results are considered, the best classification accuracy is achieved with $51.76 \%$ of accuracy at ts 80 . It is followed by $50.07 \%, 48.74 \%, 43.48 \%$ of accuracies at ts50, ts 30 , and ts 10 , respectively. At ts80, CNN as a deep learning model outperforms all traditional machine learning algorithms by reaching $58.29 \%$ of accuracy level. This means that CNN exhibits an outstanding classification performance when both conventional machine learning models and mean classification success of the system are considered. The classification performance of each model is ordered at ts 80 as CNN, RF, SVM, NB, DT with $58.29 \%, 54.45 \%, 54.22 \%, 53.70 \%, 38.15 \%$ of accuracies, respectively. This indicates that CNN has shown approximately an increase of at least $4 \%$ in classification performance compared to the best conventional machine learning classifier RF. Considering the poorest classification performance of the machine learning algorithm DT, CNN displays nearly a maximum $10 \%$ increase in classification success. If a deep learning algorithm was not included in the proposed system, the random forest method as a machine learning model would be the ideal method for this resonance frequency classification task. In Table 2, all evaluation metrics are demonstrated in order to observe the success of classifiers. In Table 3, the resonance frequency classification accuracies of each method are exhibited on dataset 2 by changing training set percentages. We observe the significant improvement when data set is extended by gathering more instances in classification accuracies of CNN model. While the best classification algorithm presents $58.29 \%$ of accuracy on dataset1, CNN performs $68.77 \%$ of accuracy at the same training set size. success for $\mathrm{CNN}$ is arisen from the extended version Approximately, 10\% enhancement in
Table 1. Classification accuracies of each model on dataset 1 in terms of training set percentages

\begin{tabular}{|c|c|c|c|c|}
\hline \multirow{2}{*}{ Models } & \multicolumn{4}{|c|}{ Training Set Percentages (ts) } \\
\cline { 2 - 5 } & $\boldsymbol{t s 8 0}$ & $\boldsymbol{t s 5 0}$ & $\boldsymbol{t s 3 0}$ & $\boldsymbol{t} \mathbf{s 1 0}$ \\
\hline CNN & 58.29 & 56.05 & 55.12 & 49.50 \\
\hline SVM & 54.22 & 53.85 & 52.46 & 47.17 \\
\hline DecTree & 38.15 & 36.56 & 35.09 & 29.00 \\
\hline NavieBayes & 53.70 & 50.11 & 49.20 & 44.82 \\
\hline $\begin{array}{c}\text { Random } \\
\text { Forest }\end{array}$ & 54.45 & 53.78 & 51.84 & 46.90 \\
\hline Average & 51.76 & 50.07 & 48.74 & 43.48 \\
\hline
\end{tabular}

Table 2. The results of evaluation metrics on datasetl at ts 80 .

\begin{tabular}{|c|c|c|c|c|}
\hline \multirow{2}{*}{ Models } & \multicolumn{4}{|c|}{ Evaluation Metrics } \\
\cline { 2 - 5 } & Accuracy & F-measure & Precision & Sensitivity \\
\hline CNN & 58.29 & 65.44 & 62.13 & 68.75 \\
\hline SVM & 54.22 & 60.85 & 57.02 & 65.80 \\
\hline DecTree & 38.15 & 33.67 & 30.33 & 38.24 \\
\hline NavieBayes & 53.70 & 59.38 & 57.25 & 63.72 \\
\hline $\begin{array}{c}\text { Random } \\
\text { Forest }\end{array}$ & 54.45 & 61.74 & 61.16 & 60.98 \\
\hline Average & 51.76 & 56.22 & 53.58 & 59.50 \\
\hline
\end{tabular}

classification of data set when the same experimental settings are taken into account. The similar classification performance order is observed on dataset2 as $\mathrm{CNN}>\mathrm{RF}>\mathrm{SVM}>\mathrm{NB}>\mathrm{DT}$ at ts80. On dataset 2 at ts 80 , a minimum of $13 \%$ enhancement and a maximum of $22 \%$ improvement is observed when the classification performances of RF and DT are considered, respectively. As a result of Table 1 and Table 3 on both data sets, CNN as a deep learning algorithm performs superior classification performance while DT as a machine learning algorithm performs the poorest resonance frequency classification success. In Table 4, all evaluation metrics are indicated to observe the performance of classifiers.

Table 3. Classification accuracies of each model on dataset 2 in terms of training set percentages

\begin{tabular}{|c|c|c|c|c|}
\hline \multirow{2}{*}{ Models } & \multicolumn{4}{|c|}{ Training Set Percentages (ts) } \\
\cline { 2 - 5 } & $\boldsymbol{t s 8 0}$ & $\boldsymbol{t s 5 0}$ & $\boldsymbol{t} \mathbf{s 3 0}$ & $\boldsymbol{t} \mathbf{s 1 0}$ \\
\hline CNN & 68.77 & 66.10 & 61.53 & 55.40 \\
\hline SVM & 54.91 & 54.05 & 47.64 & 39.00 \\
\hline DecTree & 46.42 & 45.11 & 38.88 & 32.56 \\
\hline NavieBayes & 52.20 & 50.32 & 45.09 & 37.91 \\
\hline $\begin{array}{c}\text { Random } \\
\text { Forest }\end{array}$ & 55.84 & 54.72 & 49.36 & 42.55 \\
\hline Average & 55.63 & 54.06 & 48.50 & 41.48 \\
\hline
\end{tabular}


Table 4. The results of evaluation metrics on dataset 2 at ts 80 .

\begin{tabular}{|c|c|c|c|c|}
\hline \multirow{2}{*}{ Models } & \multicolumn{4}{|c|}{ Evaluation Metrics } \\
\cline { 2 - 5 } & Accuracy & F-measure & Precision & Sensitivity \\
\hline CNN & 68.77 & 76.51 & 73.04 & 80.59 \\
\hline SVM & 54.91 & 59.27 & 56.75 & 64.82 \\
\hline DecTree & 46.42 & 40.05 & 38.27 & 47.33 \\
\hline NavieBayes & 52.20 & 57.78 & 55.15 & 65.10 \\
\hline $\begin{array}{c}\text { Random } \\
\text { Forest }\end{array}$ & 55.84 & 61.42 & 60.09 & 69.71 \\
\hline Average & 55.63 & 59.01 & 56.66 & 65.51 \\
\hline
\end{tabular}

Figure 3 demonstrates training-validation loss and training-validation accuracy values of the best model $\mathrm{CNN}$ at ts80 in terms of epoch sizes on dataset2. As the number of epoch size increases, both training loss and test loss vary up to a certain epoch value which is 80 in this study.

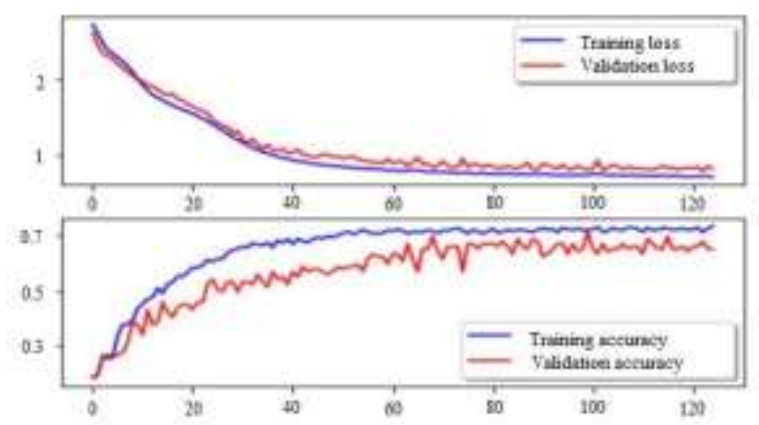

Figure 3. Training-validation loss and trainingvalidation accuracy results of CNN model at ts80 in terms of epoch size on dataset 2.

The training procedure can be stopped after this stage as no changes are observed in the subsequent increases in the number of attempts. Moreover, overfitting problem is handled employing early stopping criterion, dropout function, and L2 regularization as seen in Figure 1. This means the proposed model is trained with $\mathrm{CNN}$ method on dataset 2 without any over-fitting challenge.

\section{Conclusion and Discussion}

In this work, we concentrate on the estimation of chiral metamaterial resonance by comparing the classification performance of both conventional machine learning models and convolutional neural networks as deep learning model. For this purpose, convolutional neural networks, support vector machines, naive Bayes, decision trees, random forests are employed for the classification of resonance frequency. Comprehensive experiments are carried out by varying training set percentages, epoch sizes, and data sets. Experiment results demonstrate that the usage of $\mathrm{CNN}$ as a deep learning model exhibits superior classification success in resonance frequency compared to the traditional machine learning algorithms. Depending on the application, the resonant structure can be designed by its geometrical parameters and relative positioning in the electromagnetic field. In future, we plan to investigate the impact of various deep learning algorithms on frequency of chiral metamaterial resonance and relative importance of design parameters on the resonance frequency.

\section{Author Statements:}

- The authors declare that they have equal right on this paper.

- The authors declare that they have no known competing financial interests or personal relationships that could have appeared to influence the work reported in this paper

- The authors declare that they have nobody or nocompany to acknowledge.

\section{References}

[1] Lord Kelvin, in Baltimore Lectures on Molecular Dynamics and the Wave Theory of Light, Clay and Sons: London, 1904, p. 449.

[2] Barron, Laurence D. Molecular light scattering and optical activity. Cambridge University Press, 2004.

[3] Smith, D. R., Padilla, W. J., Vier, D. C., NematNasser, S. C., Schultz, S. "Composite medium with simultaneously negative permeability and permittivity." Physical review letters 84.18 (2000), 4184.

[4] Zhao, R., Zhang, L., Zhou, J., Koschny, T., Soukoulis, C. M. "Conjugated gammadion chiral metamaterial with uniaxial optical activity and negative refractive index." Physical Review B, 83.3 (2011): 035105.

[5] Wang, B., Zhou, J., Koschny, T., Soukoulis, C. M. "Nonplanar chiral metamaterials with negative index." Applied Physics Letters 94.15 (2009): 151112.

[6] Zhou, J., Dong, J., Wang, B., Koschny, T., Kafesaki, M., Soukoulis, C. M. "Negative refractive index due to chirality." Physical Review B 79.12 (2009): 121104.

[7] Kenanakis, G., Zhao, R., Stavrinidis, A., Konstantinidis, G., Katsarakis, N., Kafesaki, M., Economou, E. N. "Flexible chiral metamaterials in the terahertz regime: a comparative study of various designs." Optical Materials Express 2.12 (2012): 1702-1712.

[8] Zhang, S., Park, Y. S., Li, J., Lu, X., Zhang, W., Zhang, X. "Negative refractive index in chiral metamaterials." Physical review letters 102.2 (2009): 023901. 
[9] Kuwata-Gonokami, M., Saito, N., Ino, Y., Kauranen, M., Je_movs, K., Vallius, T., Svirko, Y. "Giant optical activity in quasi-two-dimensional planar nanostructures." Physical review letters 95.22 (2005): 227401.

[10] Dong, J., Zhou, J., Koschny, T., Soukoulis, C. "Bilayer cross chiral structure with strong optical activity and negative refractive index." Optics Express 17.16 (2009): 14172-14179.

[11] Marqus, R., Medina, F., Ra_i-El-Idrissi, R. "Role of bianisotropy in negative permeability and lefthanded metamaterials." Physical Review B 65.14 (2002): 144440.

[12] Marqus, R., Mesa, F., Martel, J., Medina, F. "Comparative analysis of edge-and broadsidecoupled split ring resonators for metamaterial design-theory and experiments." IEEE Transactions on antennas and propagation 51.10 (2003): 25722581.

[13] Huangfu, J., Ran, L., Chen, H., Zhang, X. M., Chen, K., Grzegorczyk, T. M., Kong, J. A. "Experimental con_rmation of negative refractive index of a metamaterial composed of -like metallic patterns." Applied Physics Letters 84.9 (2004): 1537-1539.

[14] Ran, L., Huangfu, J. T., Chen, H. S., Li, Y., Zhang, X., Chen, K., Kong, J. A. "Microwave solid- state left-handed material with a broad bandwidth and an ultralow loss." Physical Review B , 70.7 (2004): 073102.

[15] Aydin, K., Li, Z., Hudlika, M., Tretyakov, S. A., Ozbay, E. "Transmission characteristics of bianisotropic metamaterials based on omega shaped metallic inclusions." New Journal of Physics, 9.9 (2007): 326.

[16] Jaggard, D. L., Mickelson, A. R., Papas, C. H. "On electromagnetic waves in chiral media." Applied physics 18.2 (1979): 211-216.

[17] Tretyakov, S. A., Mariotte, F., Simovski, C. R., Kharina, T. G., Heliot, J. P. "Analytical antenna model for chiral scatterers: Comparison with numerical and experimental data." IEEE Transactions on Antennas and Propagation 44.7 (1996): 1006-1014.

[18] Saenz, E., Semchenko, I., Khakhomov, S., Guven, K., Gonzalo, R., Ozbay, E., Tretyakov, S. "Modeling of spirals with equal dielectric, magnetic, and chiral susceptibilities." Electromagnetics 28.7 (2008): 476493.

[19] Guven, K., Saenz, E., Gonzalo, R., Ozbay, E., Tretyakov, S. "Electromagnetic cloaking with canonical spiral inclusions." New Journal of Physics 10.11 (2008): 115037.

[20] I. Malkiel, M. Mrejen, A. Nagler, U. Arieli, L. Wolf and H. Suchowski, "Deep learning for the design of nano-photonic structures," 2018 IEEE International Conference on Computational Photography (ICCP), Pittsburgh, PA, 2018, pp. 1-14, doi: 10.1109/ICCPHOT.2018.8368462.

[21] Kiarashinejad, Yashar, et al. "Deep learning reveals underlying physics of lightmatter interactions in nanophotonic devices." Advanced Theory and Simulations 2.9 (2019): 1900088.
[22] Ma, Wei, Feng Cheng, and Yongmin Liu. "Deeplearning-enabled on-demand design of chiral metamaterials." ACS nano 12.6 (2018): 6326-6334.

[23] Yao, Kan, Rohit Unni, and Yuebing Zheng. "Intelligent nanophotonics: merging photonics and artifcial intelligence at the nanoscale." Nanophotonics 8.3 (2019): 339-366.

[24] Peurifoy, John, et al. "Nanophotonic particle simulation and inverse design using arti_cial neural networks." Science advances 4.6 (2018).

[25] Malkiel, Itzik, et al. "Plasmonic nanostructure design and characterization via deep learning." Light: Science Applications 7.1 (2018): 1-8.

[26] Ma, W., Cheng, F., Xu, Y., Wen, Q., Liu, Y. (2019). Probabilistic Representation and Inverse Design of Metamaterials Based on a Deep Generative Model with SemiSupervised Learning Strategy. Advanced Materials, 31(35), 1901111.

[27] Ahmed, Waqas W., et al. "Deterministic and probabilistic deep learning models for inverse design of broadband acoustic cloak." Physical Review Research 3.1 (2021): 013142.

[28] Huang, Wei, et al. "Inverse engineering of electromagnetically induced transparency in terahertz metamaterial via deep learning." Journal of Physics D: Applied Physics 54.13 (2021): 135102.

[29] Tao, Zilong, et al. "Optical circular dichroism engineering in chiral metamaterials utilizing a deep learning network." Optics Letters 45.6 (2020): 1403-1406.

[30] Lininger, Andrew, Michael Hinczewski, and Giuseppe Strangi. "General Inverse Design of ThinFilm Metamaterials with Convolutional Neural Networks." arXiv preprint arXiv:2104.01952 (2021).

[31] McCallum, A., Nigam, K." A comparison of event models for naive bayes text classification." In AAAI-98 workshop on learning for text categorization (Vol. 752, No. 1, pp. 41-48),1998.

[32] Manning, C., Schutze, H. "Foundations of statistical natural language processing." MIT press, 1999.

[33] Kilimci, Z. H., Gven, A., Uysal, M., Akyokus, S." Mood detection from physical and neurophysical data using deep learning models." Complexity, 2019.

[34] Kilimci, Z. H., Omurca, S. I." Extended feature spaces based classifier ensembles for sentiment analysis of short texts." Information Technology and Control, 47(3), 457-470, 2018.

[35] Kilimci, Z. H., Akyokus, S." Deep learning-and word embedding-based heterogeneous classifier ensembles for text classification." Complexity, 2018.

[36] Kilimci, Z. H., Ganiz, M. C. "Evaluation of classification models for language processing." In 2015 International Symposium on Innovations in Intelligent SysTems and Applications (INISTA) (pp. 1-8). IEEE, 2015.

[37] Joachims T. "Text Categorization with Support Vector Machines: Learning with Many Relevant Features." In: 10th European Conference on Machine Learning; 1998; Chemnitz, Germany: pp.137-142. 
[38] Burges CJC." A Tutorial on Support Vector Machines for Pattern Recognition." In: 3rd International Conference on Knowledge Discovery and Data Mining; 1998; New York, USA: pp. 121167.

[39] Yang Y, Liu X. "A Re-examination of Text Categorization Methods." In: 22nd Annual nternational ACM SIGIR Conference on Research and Development in Information Retrieval; 1999; Berkeley, CA, USA: pp. 42-49.

[40] Kilimci, Z. H., Akyuz, A. O., Uysal, M., Akyokus, S., Uysal, M. O., Atak Bulbul, B., Ekmis, M. A." An improved demand forecasting model using deep learning approach and proposed decision integration strategy for supply chain." Complexity, 2019.

[41] Quinlan, J. R. (1986)."Induction of decision trees. Machine learning." 1(1), 81-106.

[42] Kilimci, Z. H., Akyokus, S. (2019, July). "The analysis of text categorization represented with word embeddings using homogeneous classifiers." In 2019 IEEE International Symposium on Innovations in Intelligent SysTems and Applications (INISTA) (pp. 1-6). IEEE.

[43] Kilimci, Z. H., Omurca, S. I. (2017, August). "A Comparison of Extended Space Forests for Classifier Ensembles on Short Turkish Texts." In International Academic Conference on Engineering, IT and Artificial Intelligence (pp. 96-104).

[44] L. Breiman, "Random forests." Machine Learning, vol. 45, no. 1, pp. 532, 2001.

[45] Kilimci, Z. H., Akyokus, S., Omurca, S. I. (2016, August). "The effectiveness of homogenous ensemble classifiers for Turkish and English texts." In 2016 International Symposium on Innovations in Intelligent SysTems and Applications (INISTA) (pp. 1-7). IEEE.

[46] Kilimci, Z. H., Akyokus, S., Omurca, S. . (2017, July). "The evaluation of heterogeneous classifier ensembles for Turkish texts." In 2017 IEEE International Conference on Innovations in Intelligent SysTems and Applications (INISTA) (pp. 307-311). IEEE.

[47] Y. Lecun, L. Bottou, Y. Bengio, and P. Ha_ner, "Gradientbased learning applied to document recognition." Proceedings of the IEEE, vol. 86, no. 11, pp. 22782324, 1998.

[48] J. Schmidhuber, "Deep learning in neural networks: an overview." Neural Networks, vol. 61, pp. 85117, 2015.

[49] Y. LeCun, Y. Bengio, and G. Hinton, "Deep learning." Nature, vol. 521, no. 7553, pp. 436444, 2015.

[50] Tanberk, S., Kilimci, Z. H., Tkel, D. B., Uysal, M., Akyoku, S. "A Hybrid Deep Model Using Deep Learning and Dense Optical Flow Approaches for Human Activity Recognition." IEEE Access, 8, 19799-19809, 2020.

[51] Kilimci, Z. H., Akyokus, S." The evaluation of word embedding models and deep learning algorithms for Turkish text classification." In 2019 4th International Conference on Computer Science and Engineering (UBMK) (pp. 548-553). IEEE.
[52] Kilimci, Z. H. "Sentiment Analysis Based Direction Prediction in Bitcoin using Deep Learning Algorithms and Word Embedding Models." International Journal of Intelligent Systems and Applications in Engineering, 8(2), 60-65, 2020.

[53] Kilimci, Z. H. "Borsa tahmini için Derin Topluluk Modelleri (DTM) ile finansal duygu analizi." Journal of the Faculty of Engineering Architecture of Gazi University, 35(2), 635-650, 2020.

[54] Cevik, F., Kilimci, Z. H."The evaluation of Parkinson's disease with sentiment analysis using deep learning methods and word embedding models." Pamukkale University Journal of Engineering Sciences, 27(2), 151-161, 2021.

[55] Othan, D., Kilimci, Z. H., Uysal, M." Financial Sentiment Analysis for Predicting Direction of Stocks using Bidirectional Encoder Representations from Transformers (BERT) and Deep Learning Models." In Proc. Int. Conf. Innov. Intell. Technol., vol. 2019, pp. 30-35, 2019. 